\title{
A Comparison of CNN and LEGION Networks
}

\author{
DeLiang Wang \\ Department of Computer Science and Engineering \\ and Center for Cognitive Science \\ The Ohio State University, Columbus, Ohio 43210, USA \\ Email: dwang@cis.ohio-state.edu
}

\begin{abstract}
CNN and LEGION networks have been extensively studied in recent years. These two frameworks share many common features; both employ continuous-time dynamics, are nonlinear, and emphasize local connectivity. In addition, they both have been successfully applied to visual processing tasks and implemented on analog VLSI chips. This paper investigates the relations between the two frameworks. We present their standard versions, and contrast the underlying dynamics and connectivity. We also describe several tasks where both CNN and LEGION have been applied. The comparison reveals fundamental differences between them. CNN is good for early visual processing, whereas LEGION is good for midlevel visual processing. Furthermore, the comparison suggests that a combined network will likely enhance the overall processing capability.
\end{abstract}

\section{INTRODUCTION}

Two types of neural network have been extensively studied in recent years. The first type is CNN (cellular neural network ${ }^{*}$ ) [6] [5], and the second type is LEGION (locally excitatory globally inhibitory oscillator network) [16] [22]. There are many common features between CNN and LEGION. Chief among them are continuous-time dynamics and nonlinearity. From the standpoint of network architecture, they are the same except that LEGION includes, in addition to local connectivity, a global inhibitor. These features make them well suited for direct circuit implementation, and indeed they have been. In addition, both have been successfully applied to visual processing tasks. Yet, they were originated with different motivations and for different purposes, and their developments proceed along largely independent paths. The similarities between the two frameworks arouse interesting questions of whether $\mathrm{CNN}$ and LEGION have deeper connections between one another and in what ways they are similar or different.

In an attempt to address these questions, this paper presents the standard versions of CNN and LEGION, and compares their underlying dynamics and coupling schemes. To further illuminate their relations, I describe several common tasks that both $\mathrm{CNN}$ and LEGION have been

\footnotetext{
* CNN may also stand for Cellular Nonlinear Network [4].
}

successfully applied. Our comparative study reveals that there are fundamental differences between these two networks, and they are good for different informationprocessing tasks. Their relative strengths and weaknesses are contrasted with respect to visual processing. Furthermore, the comparison suggests that CNN is well suited for early visual processing whereas LEGION is well suited for midlevel processing, and a combination of the two should significantly strengthen the overall processing capability.

\section{DEFINITIONS AND BASIC PROPERTIES OF CNN AND LEGION}

Largely motivated by computational capabilities of Hopfield networks, Chua and Yang in 1988 [6] [5] proposed CNN to circumvent the full connectivity requirement of a Hopfield network, which is impractical for VLSI circuit implementation. Recall that in a Hopfield network, each unit is connected with all the units of the network, leading to the so-called "curse of interconnecting wires" from the VLSI implementation perspective [4]. A CNN network allows only local connections between network units, forming a cellular structure in the sense of cellular automata.

On the other hand, LEGION was originally proposed by Terman and Wang in 1995 [16] [22] to address the binding problem in both natural and artificial neural networks, which refers to how sensory elements organize into perceived objects or how the responses of local feature-detecting neurons in different areas of the brain are bound together to form global percepts. A LEGION network is a network of neural oscillators with local excitatory connections between oscillators and global inhibition via a global inhibitor.

The following gives detailed definitions of $\mathrm{CNN}$ and LEGION. Here, I do not aim at mathematical generality, which can often be obtained by, for example, substituting parameters for constants. Rather, I highlight the commonality between the two models.

\section{A. $C N N$}

The building block of CNN is a single cell $C_{i j}$, where the subscript indexes the cell location on a two-dimensional (2- 
D) network* ${ }^{*}$ The state variable of $C_{i j}$ is defined by the following linear differential equation:

$$
\dot{x}_{i j}=-x_{i j}+I_{i j}+\sum_{k l \in N(i, j)} A_{k l} y_{k l}+\sum_{k l \in N(i, j)} B_{k l} u_{k l}
$$

where $I_{i j}$ indicates a threshold of the cell and $N(i, j)$ represents the set of neighboring cells that connect to $C_{i j}$, where 4 nearest-neighbor or 8 nearest-neighbor connectivity is often chosen. The output equation is given by the following function:

$$
y_{i j}=\frac{1}{2}\left(\left|x_{i j}+1\right|-\left|x_{i j}-1\right|\right)=\left\{\begin{array}{cc}
1, & x_{i j} \geq 1 \\
x_{i j}, & 1 \geq x_{i j} \geq-1 \\
-1, & x_{i j} \leq-1
\end{array}\right.
$$

which is a piecewise linear function resembling a sigmoid. The piecewise linear output function is the origin of nonlinearity in $\mathrm{CNN}$ models. In addition, the corresponding external input is denoted by $u_{i j}$. The $\mathbf{A}$ matrix specifies the connectivity between cells, and is called feedback template. The B matrix specifies the connectivity from external input to network cells, and is called the control template. The two matrices, together with Iij, completely define a CNN network.

Chua and Yang [6] analyzed the stability of CNN networks, and showed that CNN cells approach stable equilibrium points under certain conditions, most notably that the A template is symmetric with respect to its center. In other words, a CNN network does not oscillate or become chaotic under these conditions. This symmetry requirement on feedback connectivity is akin to that of Hopfield networks in order to guarantee network stability. Another interesting result is that, if the center element of $\mathbf{A}$ is greater than 1, the output value of each $\mathrm{CNN}$ cell will be bipolar, i.e. either 1 or -1 , after the network reaches an equilibrium point.

\section{B. LEGION}

The development LEGION was directly motivated by the discovery of coherent oscillations in the visual cortex in the late 1980s. LEGION owes its conceptual foundation to the oscillatory correlation theory [17] [16], where feature detectors are represented by oscillators and binding is represented by synchrony within an assembly of oscillators and desynchrony between different assemblies. The building block of LEGION is a single relaxation oscillator, $O_{i j}$, defined by an excitatory variable $x_{i j}$ and an inhibitory variable $y_{i j}$ :

\footnotetext{
* It is straightforward to extend to dimensions other than 2-D.
}

$$
\begin{aligned}
& \dot{x}_{i j}=3 x_{i j}-x_{i j}^{3}+2-y_{i j}+u_{i j}+S_{i j}+\rho \\
& \dot{y}_{i j}=\varepsilon\left(\alpha\left(1+\tanh \left(x_{i j} / \beta\right)\right)-y_{i j}\right)
\end{aligned}
$$

Here, $u_{i j}$ denotes external stimulation to $O_{i j}$ and $S_{i j}$ the overall coupling from the rest of the network. $\rho$ indicates a noise term, introduced to assist in desynchronization. $\varepsilon$ is a small positive number, and $\alpha$ are $\beta$ parameters. When coupling and noise are ignored and $u$ is set to a constant, (3) defines a typical relaxation oscillator with two time scales induced by $\varepsilon$. The $x$-nullcline (i.e. $\dot{x}_{i j}=0$ ) is a cubic function and the $y$-nullcline is a sigmoid function; these represent the nonlinearity of the system.

If $u>0, O_{i j}$ produces a stable limit cycle and is called enabled. The limit cycle alternates between a phase of relatively high $x$ values and a phase of relatively low $x$ values, called the active and silent phase respectively. Within each of the two phases the oscillator exhibits near steady-state behavior. In contrast to the behavior within each phase, the transition between the two phases takes place rapidly, and it is referred to as jumping. If $u<0$, no oscillation occurs and $O_{i j}$ is called excitable. Obviously, whether an oscillator is enabled or excitable depends on external stimulation. Hence, oscillations in (3) are stimulusdependent.

For a 2-D LEGION network, the coupling term $S_{i j}$ in (3) is given by

$$
S_{i j}=\sum_{k l \in N(i, j)} W_{k l} H\left(x_{k l}\right)-W_{z} H\left(z-\theta_{z}\right)
$$

where $H$ stands for the Heaviside step function, $W_{k l}$ is the connection weight from oscillator $O_{k l}$ to $O_{i j}$, and $N(i, j)$ is as defined in (1). The parameter $\theta_{z}$ is a threshold, and $W_{z}$ is the weight of inhibition from the global inhibitor $z$, defined as

$$
\dot{z}=\phi\left(\sigma_{\infty}-z\right)
$$

Here, $\phi$ is a parameter, and $\sigma_{\infty}=1$ if $x_{i j} \geq \theta_{z}$ for at least one oscillator $O_{i j}$ and $\sigma_{\infty}=0$ otherwise. If $\sigma_{\infty}$ equals $1, z \rightarrow 1$.

As shown by Terman and Wang [16], a LEGION network exhibits the mechanism of selective gating, where an enabled oscillator jumping up to the active phase rapidly recruits the oscillators stimulated by the same pattern (a connected region) while preventing others from jumping up. They proved that, due to selective gating, the network rapidly achieves both synchronization within each oscillator assembly and desynchronization between different assemblies. Desynchronization between two assemblies means that they are never active simultaneously. In addition, the overall time the system takes to achieve both 
synchronization and desynchronization is no greater than $m$ cycles of oscillations, where $m$ is the number of patterns in the input image. See [19] for a tutorial exposition of the selective gating mechanism and other related properties of relaxation oscillators and their networks.

\section{EXAMPLE APPLICATIONS}

In order to illustrate similarities and differences between CNN and LEGION processing, I review several informationprocessing tasks where $\mathrm{CNN}$ and LEGION have been applied.

\section{A. Early Visual Processing}

CNN has been applied to a number of local visual processing tasks, such as edge detection, corner detection, image translation from one position to another, etc., by taking advantage of single cell computation and local activity propagation due to the use of a feedback template [4] [8]. Though such tasks have not been explicitly addressed in the LEGION framework, it should be straightforward to apply LEGION to them.

A more interesting task $\mathrm{CNN}$ can perform is image smoothing: Removing noise on an image and preserving major image features [14]. Perona and Malik [13] showed that one can perform smoothing by anisotropic diffusion, which is a task naturally suited for the CNN framework because a CNN system essentially realizes a reactiondiffusion process. The diffusion process through local feedback connectivity can propagate information globally through time. This is similar to image smoothing via a Gaussian kernel. But, as pointed out by Perona and Malik, diffusion via a dynamical system can achieve image smoothing at multiple scales in a scale space, whereas a spatial filter (such as a Gaussian kernel) performs image smoothing at a fixed scale.

Image smoothing has also been studied in the LEGION framework by Chen et al. [3]. They use weight adaptation to accomplish feature-preserving smoothing, and demonstrate that their nonlinear smoothing technique overcomes several difficulties in other smoothing methods, including anisotripic diffusion. One such difficulty is the so-called termination problem: When does the system stop the diffusion process, since the process, unstopped, eventually leads to a constantintensity image? Due to the intrinsic connection to anisotropic diffusion, CNN networks suffer from similar difficulties.

Strictly speaking, the weight adaptation technique proposed by Chen et al. [3] is not an intrinsic part of LEGION. On the other hand, locally-coupled spike oscillator networks have been successfully applied to image smoothing [9] [10]. The basic unit of such a network is a spike oscillator, or a spiking neuron model, which has an instantaneous active phase in comparison with a relaxation oscillator. Campbell et al. [1] recently showed that the basic relaxation oscillator equation of (3) can exhibit qualitatively different kinds of oscillation, including spike oscillation and sinusoidal oscillation, through appropriate parameter choices. This implies that a LEGION network can perform image smoothing in the same way as a spike oscillator network. Another way of relating LEGION to $\mathrm{CNN}$ is through the use of sinusoidal oscillations, where oscillators communicate with each other directly in oscillation phase, a continuous variable. Sinusoidal (phase) oscillator networks have not been studied for image smoothing, but the above connections suggest that they hold strong promise for such visual processing.

\section{B. Connectedness Detection}

A classic problem in neural networks is the Minsky and Papert connectedness problem: How to tell whether an input figure is connected [12]. They showed that this problem cannot be solved by simple or multilayer perceptrons, which are feedforward networks. Both $\mathrm{CNN}$ and LEGION have been shown to be able to solve this problem.

When a binary input figure is presented to a CNN network, where each pixel is mapped to a single cell, the network can solve the connectedness problem in the following way [4]. First, a cell triggered by any given black pixel (high input) deactivates itself and its neighboring cells, which further deactivate themselves and their neighbors, and so on. The propagation of deactivation ultimately deactivates an entire connected pattern. If the figure contains a single connected pattern, or is connected, then the entire network will be deactivated after the propagation. Otherwise, the input figure is disconnected, or it contains more than one connected pattern.

LEGION solves the connectedness problem in a different way [20]. The selective gating mechanism ensures that all the oscillators representing a single connected pattern are synchronized while those representing different connected patterns are desynchronized. The global inhibitor oscillates with a frequency that is as many times that of single enabled oscillators as the number of connected patterns in the input figure. Thus, how many patterns are in the input figure is revealed by the ratio of the frequency of the global inhibitor to the oscillation frequency of an enabled oscillator [20]. A ratio of 1 indicates that the input figure contains one pattern, and thus the figure is connected. A ratio greater than 1 indicates that the input contains more than one pattern and therefore the figure is disconnected.

Besides computing the connectedness predicate, the LEGION solution also counts how many connected patterns are in the input figure. This counting function is not easily done by a CNN network, although one could apply the deactivation process iteratively. Such an operation, however, seems to require processing external to $\mathrm{CNN}$ computing. 


\section{Image Segmentation}

Unlike early visual processing that can be performed primarily via local operations, image segmentation, or perceptual organization, belongs to midlevel processing which takes into consideration of global image structure. Nonetheless, CNN has been applied to image segmentation.

Stoffel et al. [14] studied CNN-based image segmentation. Their method decomposes the problem into multiple processing stages, each of which is implemented by a CNN network tailored for the task. These stages include image smoothing, edge detection, and major feature extraction through simple morphological operations. After these stages are performed, the segmentation network then performs contour extraction and hollow filling in order to fill in small fragments and merge them with major regions. The result of segmentation is a collection of closed contours. Their method has been applied to intensity- and motionbased segmentation. Subsequent work has also addressed texture image segmentation [15].

Since a direct motivation for inventing LEGION is to address the binding problem, which is essentially the segmentation problem, it is not surprising that LEGION has been applied to a variety of image segmentation tasks. To deal with real-world images, a LEGION network needs to encode a measure of similarity between local image elements, which may be pixel values for intensity images or statistical features that characterize a textural pattern. The basic approach to image segmentation by LEGION is the following. After a scene is presented, feature extraction first takes place, and extracted features form the basis for determining connection weights between oscillators. The oscillator network then evolves autonomously. After a number of oscillation cycles required for the synchronization and desynchronization process, oscillator assemblies are formed and they represent resulting segments. Different segments emerge from the network at different times, and it is segmentation in time that distinguishes this approach from others. LEGION has been successfully applied to intensity, range, motion, and texture images (see [21] for a review).

Because the encoding of an image in a LEGION network and the LEGION segmentation mechanism have a higher degree of flexibility, and segmentation results by LEGION are better than those by CNN. For instance, a same image ("Claire") has been used in both CNN [14] and LEGION motion segmentation [23] (see also [2]), and the latter produces significantly better results (e.g. the whole face is segmented as a single region). On the other hand, added flexibility in LEGION segmentation may complicate VLSI implementation (see Sect. IV.D).

\section{GENERAL COMPARISONS}

\section{A. Unit Dynamics}

The most basic difference between CNN and LEGION lies in the dynamics of single units. In $\mathrm{CNN}$, unit dynamics is equilibrium dynamics like that of its predecessor - the Hopfield network. In contrast, LEGION dynamics is oscillatory dynamics; LEGION was proposed for the purpose of overcoming limitations of equilibrium dynamics. Oscillations offer an extra degree of freedom: phase, in comparison to steady states. The oscillatory correlation theory employs oscillation phase to encode the binding relationship between feature detectors. Note that, in addition to phase an oscillator can represent its overall level of activity by oscillation frequency, analogous to neuronal activity.

Lacking the dimension of phase seems a fundamental limitation of CNN. Because of this limitation, $\mathrm{CNN}$ networks have trouble representing multiple objects on an image simultaneously. This is hinted in Sect. III.B, where solutions to the connectedness problem are discussed. CNN has no trouble representing the binary state of whether a figure is connected, but has difficulty in representing the number of connected patterns in a figure. This difficulty persists when it is applied to segmenting multiple regions of an image. Even if one could imagine a CNN network that produces graded output levels instead of (2), how to read such outputs is likely a tricky issue (see related discussions in [21]).

\section{B. Connectivity}

Both CNN and LEGION possess local feedback connections. Additionally LEGION has global inhibition via connections between individual oscillators and the global inhibitor. The role of the global inhibitor is to desynchronize multiple oscillator assemblies. It is also a vehicle for global communication in a LEGION network. Such global interaction is, for example, important for counting the number of connected patterns (Sect. III.B) and for objectlevel attentional selection.

The motivation for $\mathrm{CNN}$ local connectivity is to facilitate circuit implementation, or to combat the curse of interconnecting wires. The local connectivity in $\mathrm{CNN}$ has turned out to be very fruitful for circuit implementation (see Sect. IV.D). LEGION is primarily locally connected; the number of connections caused by the global inhibitor is only a fraction of that of local connections between oscillators. Hence in terms of circuit implementation, such connections do not seem to constitute a major issue.

\section{Visual Processing}

As discussed in Sect. III.B, CNN is suited for local operations on an input image, and such operations correspond to early visual processing. CNN has also been applied to computing individual processing stages for midlevel processing tasks such as image segmentation. Decomposing a complex task into component tasks is a sensible information-processing strategy. But the decomposition process inevitably introduces human intervention. If the goal is to perform autonomous 
computation, CNN networks are likely limited in performing larger, challenging tasks autonomously.

On the other hand, LEGION has been mainly applied to midlevel scene segmentation tasks. Such a task is generally preceded by a local feature extraction stage, which analyzes color, motion, depth, texture, etc. Though such feature extraction has been addressed in conjunction with LEGION image segmentation, feature extraction should be viewed as separate from grouping and segmentation by oscillatory correlation; feature extraction belongs to the realm of early visual processing.

The above analysis logically suggests that CNN is better suited for early visual processing and LEGION is better suited for midlevel visual processing. For a visual analysis task, early and midlevel, as well as high-level processing all take place in the visual system to accomplish the task. This points to synergy between CNN and LEGION processing, and combining them in performing the same challenging task should enhance the overall performance.

\section{VLSI Implementation}

Circuit implementation is the principal motivation of CNN networks, hence, not surprisingly, many successful attempts have been made in VLSI implementation of CNN networks. Direct VLSI implementation has the advantages of higher processing speed, less power consumption, and less (silicon) area occupation [4], in comparison to implementation or simulation on a general-purpose digital computer. Many tasks have been processed on CNN chips; most of them perform local image processing. Several studies also attempt to implement image segmentation tasks, which generally need a hybrid of analog circuits and digital operations. For example, a recent study implements texture classification and segmentation on a $64 \times 64$ chip [15].

Much less effort has been made on circuit implementation of LEGION, partly because much of VLSI work is currently focussed on early visual processing tasks. Another reason is that nonlinear relaxation oscillators are more difficult to implement. Nonetheless, several studies have attempted to implement LEGION network on VLSI chips. For example, a recent study by Cosp and Madrenas [7] employs a hysteresis current comparator and a damped integrator to implement a single relaxation oscillator. On the basis of this, they successfully fabricated a chip implementing a 16x16 LEGION network using CMOS technology, and evaluated the chip on a number of segmentation tasks.

\section{DISCUSSION}

In 1982 Marr [11] proposed the revelational three-level analysis of complex information processing, in particular visual processing, namely, computational-theory level, representation/algorithm level, and implementation level. According to this approach, one must first perform the computational-theory analysis of a particular task before embarking on algorithm design and hardware implementation. Computational theory analysis is to clarify the goals of the computation, analyze the appropriateness of the goals, and decide on general strategies for reaching the goals. In a sentence, computational-theory analysis is to understand the character of the task. For complex tasks, this analysis is extremely important.

In essence, $\mathrm{CNN}$ is an implementation theory; a $\mathrm{CNN}$ model directly lends itself to circuit implementation. On the other hand, CNN studies do not get at computational theory analysis, nor do they get at the representation/algorithm level, at least not in a direct sense. A clear example is offered by image smoothing using anisotropic diffusion, which was proposed as an algorithm prior to its CNN implementation (see Sect. III.A). Although LEGION is motivated by representational and algorithmic considerations, it too is largely an implementation theory. Realizing this helps to clarify their roles in overall information processing. This suggests that, although CNN and LEGION studies are an integral part of modeling visual processing, they are no substitute for research at the computational-theory and algorithm levels.

The boundary between the algorithm level and implementation level becomes uniquely fuzzy in the neural networks field. Clearly one can come up with new algorithms while designing a new network for solving a particular problem. One may also treat neural network solutions as a different kind of algorithm - the parallel and distributed processing kind. LEGION image segmentation exemplifies such a situation. Indeed many researchers in neural networks unconsciously carry out both algorithm and implementation analyses together. Despite this, Marr's distinction remains a key guide for analyzing an informationprocessing task.

Building on the CNN successes, Chua proposed the concept of local activity and elevated it to the philosophical level [4]. It claims that broad phenomena of complexity, such as cooperative and competitive phenomena, far-fromthermodynamic equilibrium phenomena, and edge of chaos, cannot emerge unless the medium is locally active. The argument is established by contrasting locally active and locally passive media. Unfortunately, it says little about the sufficiency of local activity. There is no doubt that locally coupled networks can perform a wide variety of tasks. Are they adequate, say, for visual processing? Probably not. A task alluded to earlier is attentional processing, where all the neural models proposed so far employ some form of global connectivity (see [18]). Global connections also exist in the brain; for example, part of the thalamus connects with the cortex in a global way.

To conclude, I have compared CNN and LEGION networks. This comparison shows that the two frameworks are similar in many ways. Their main differences lie in the underlying unit dynamics and whether global connectivity is allowed. The comparison also suggests that $\mathrm{CNN}$ is better 
suited for early visual processing whereas LEGION is better suited for midlevel visual processing. Due to their synergistic relationship an appropriate combination of $\mathrm{CNN}$ and LEGION will likely produce significantly enhanced performance.

\section{ACKNOWLEDGMENTS}

This work was supported in part by an AFOSR grant (F49620-01-1-0027) and an NSF grant (IIS-0081058).

\section{REFERENCES}

[1] S.R. Campbell, D.L. Wang, and C. Jayaprakash, "Synchronization rates in classes of relaxation oscillators," IEEE Trans. Neural Net., in press, 2004.

[2] E. Cesmeli and D.L. Wang, "Motion segmentation based on motion/brightness integration and oscillatory correlation," IEEE Trans. Neural Net., vol. 11, pp. 935-947, 2000.

[3] K. Chen, D.L. Wang, and X. Liu, "Weight adaptation and oscillatory correlation for image segmentation," IEEE Trans. Neural Net., vol. 11, pp. 1106-1123, 2000.

[4] L.O. Chua, CNN: A paradigm for complexity. Singapore: World Scientific, 1998.

[5] L.O. Chua and L. Yang, "Cellular neural networks: Applications," IEEE Trans. Cir. Syst., vol. 35, pp. 1273-1290, 1988.

[6] L.O. Chua and L. Yang, "Cellular neural networks: Theory," IEEE Trans. Cir. Syst., vol. 35, pp. 1257-1272, 1988.

[7] J. Cosp and J. Madrenas, "Scene segmentation using neuromorphic oscillatory networks," IEEE Trans. Neural Net., vol. 14, pp. 12781296, 2003.

[8] R.C. Galan, et al., "Bio-inspired two-layer mixed-signal flexible programmable chp for early vision," IEEE Trans. Neural Net., vol. 14, pp. 1313-1336, 2003.

[9] J.J. Hopfield and A.V.M. Herz, "Rapid local synchronization of action potentials: Toward computation with coupled integrate-and-fire oscillator neurons," Proc. Natl. Acad. Sci. USA, vol. 92, pp. 6655$6662,1995$.

[10] G. Kuntimad and H.S. Ranganath, "Perfect image segmentation using pulse coupled neural networks," IEEE Trans. Neural Net., vol. 10, pp. 591-598, 1999.

[11] D. Marr, Vision. New York: Freeman, 1982.

[12] M.L. Minsky and S.A. Papert, Perceptrons. Cambridge MA: MIT Press, 1969.

[13] P. Perona and J. Malik, "Scale-space and edge detection using anisotropic diffusion," IEEE Trans. Pattern Anal. Machine Intell., vol. 12, pp. 629-639, 1990.

[14] A. Stoffels, T. Roska, and L. Chua, "Object-oriented image analysis for very-low-bitrate video-coding systems using the CNN universal machine," Int. J. Circ. Ther. Appl., vol. 25, pp. 235-258, 1997.

[15] T. Sziranyi, "Texture segmentation by the $64 \times 64$ CNN chip," in Proceedings of IEEE Workshop on CNN and Their Applications, pp. 547-554, 2002.

[16] D. Terman and D.L. Wang, "Global competition and local cooperation in a network of neural oscillators," Physica D, vol. 81, pp. 148-176, 1995.

[17] C. von der Malsburg and W. Schneider, "A neural cocktail-party processor," Biol. Cybern., vol. 54, pp. 29-40, 1986.

[18] D.L. Wang, "Object selection based on oscillatory correlation," Neural Net., vol. 12, pp. 579-592, 1999.

[19] D.L. Wang, "Relaxation oscillators and networks," in Encyclopedia of electrical and electronic engineers, J. Webster, Ed., New York: Wiley, pp. 396-405 (available on the web at www.cis.ohio-state.edu/ dwang), 1999.
[20] D.L. Wang, "On connectedness: a solution based on oscillatory correlation," Neural Comp., vol. 12, pp. 131-139, 2000.

[21] D.L. Wang, "The time dimension for neural computation," Tech. Rep. TR21, OSU Department of Computer \& Information Science, 2002 (available on the web at www.cis.ohio-state.edu/ dwang).

[22] D.L. Wang and D. Terman, "Locally excitatory globally inhibitory oscillator networks," IEEE Trans. Neural Net., vol. 6(1), pp. 283-286, 1995.

[23] X. Zhang and A.A. Minai, "Temporally sequenced intelligent blockmatching and motion-segmentation using locally coupled networks," IEEE Trans. Neural Net., in press, 2004. 